\title{
El gesto es parte del discurso y apoya el aprendizaje'
}

\author{
Juan Carlos \\ Valderrama Cárdenas ${ }^{2}$ \\ juan.valderrama@uniminuto.edu \\ Tatiana Guerrero 3 \\ tguerreropa@uniminuto.edu.co \\ Leidy Vanessa \\ AlarcónLesmes ${ }^{4}$ \\ lalarconles@uniminuto.edu.co
}

Ana Graciela

Cifuentes Gualtero 5

acifuente16@uniminuto.edu.co

Karen Nathaly

Rodríguez Cortes ${ }^{6}$

krodrigu177@uniminuto.edu.co

Leidy Carolina

Romero Quiroga7

Entrevista recibida 31/03/2020 y aprobada 21/04/2020.

\section{Cómo citar este artículo:}

Alarcón Lesmes, L. V., Cifuentes Gualtero, A. G., Guerrero, T., Rodríguez Cortes, K. N., Romero Quiroga, L. C., \& Valderrama Cardenas, J. C. (2020). El gesto es parte del discurso y apoya el aprendizaje. TransPasando Fronteras, (16). https://doi.org/10.18046/retf.i16.4163

\footnotetext{
1 Producto derivado del proyecto Alineación interactiva del semillero de investigación: Comunicación en sistemas humanos. Corporación Universitaria Minuto de Dios, programa de psicología UVD.

2 Psicólogo. Docente líder del semillero de investigación: Comunicación en sistemas humanos.

${ }^{3}$ Estudiante del programa de psicología UVD. Corporación Universitaria Minuto de Dios.

${ }^{4}$ Estudiante del programa de psicología UVD. Corporación Universitaria Minuto de Dios.

${ }^{5}$ Estudiante del programa de psicología UVD. Corporación Universitaria Minuto de Dios.

${ }^{6}$ Estudiante del programa de psicología UVD. Corporación Universitaria Minuto de Dios.

${ }^{7}$ Estudiante del programa de psicología UVD. Corporación Universitaria Minuto de Dios.
} 


\section{Resumen}

El gesto tiene un rol en la comunicación y en el aprendizaje. Además, existen mecanismos de su funcionamiento que aún están en estudio. El presente documento es el producto de la reflexión sobre las acciones corporeizadas de la comunicación de profesores y estudiantes en el aula. Para iniciar, se hace una contextualización teórica del campo de estudio, iniciando con el paradigma del comportamiento no-verbal y finalizando con la postura que supera la noción de lenguaje no-verbal. Con el propósito de comprender algunos de sus adelantos, se hizo una revisión con base en investigaciones empíricas que relacionan el gesto con procesos de aprendizaje y en cinco extractos del corpus de datos del proyecto Alineación Interactiva. Los resultados se construyeron de acuerdo con las convenciones de Gail Jefferson para la transcripción y de Mondada para el análisis de interacciones sociales en video. En conclusión, se evidencia ventajas del uso de gestos en términos de mecanismos asociados a laatención, el lenguajey la memoriaque sugieren la posibilidad de la implementación de acciones corporeizadas como parte de las estrategias pedagógicas. La reflexión tiene implicaciones prácticas para hablantes y oyentes en el aula.

Palabras clave: gesto, aprendizaje, comunicación, educación, lenguaje 


\title{
Gesture is part of speech and supports learning
}

\begin{abstract}
Gesture has a role in communication and learning. In addition, there are mechanisms of its functioning that are still under study. This document is the product of reflection on the embodied actions of communication of teachers and students in the classroom. To begin with, there is a theoretical contextualization of the field of study, beginning with the paradigm of non-verbal behavior and ending with the posture that surpasses the notion of non-verbal language. In order to understand some of its advances, a review was made based on empirical research that relates gesture to learning processes and on five extracts from the data corpus of the Interactive Alignment project. The results were constructed according to Gail Jefferson's conventions for transcription and Mondada's for the analysis of social interactions on video. In conclusion, there is evidence of advantages of the use of gestures in terms of mechanisms associated with attention, language and memory that suggest the possibility of implementing embodied actions as part of pedagogical strategies. Reflection has practical implications for speakers and listeners in the classroom.
\end{abstract}

Key words: gesture, learning, communication, education, language 


\section{Introducción}

El presente artículo hace una reflexión acerca de aspectos comunicacionales que se ven implicados en la educación y en los procesos de aprendizaje, teniendo en cuenta tanto a docentes como estudiantes que son partícipes de la relación. Para ese propósito, el artículo se construyó con base en algunas propuestas teóricas y cinco extractos del corpus teórico del proyecto de investigación Alineación Interactiva presentados con la adaptación de la convención de Jefferson para la transcripción (Follari, 2015) y la convención para interacciones sociales en video de Mondada (2016).

Toda interacción humana se hace momento-a-momento con base en mensajes que son transmitidos con la intención de comunicar y de establecer un terreno común que permita la comprensión. Al respecto, hay todo un repertorio de actos automáticos e inconscientes que están presentes en una conversación. Cuando la comunicación se hace de manera consciente se relaciona con acciones como elegir las palabras adecuadas de acuerdo con el mensaje que se quiere transmitir, es recurrente que se elijan espacios y un momento adecuado para tener una conversación exitosa. Esto llevó a establecer una distinción inicial, que se conoce como lo verbal y lo no-verbal en la comunicación.

En cuanto a la dimensión verbal, el análisis de contenido ha sido relacionado con estados psicológicos (Viney, 1983); el análisis del discurso ha tenido un nicho en los estudios políticos (Van Dijk, 2000). Incluso, es recurrente encontrar estudios sobre la comunicación verbal aplicada en diversos contextos como: prácticas relacionadas con la docencia universitaria (Ridao Rodrigo, 2017); la psicoterapia y cómo usar el lenguaje para el cambio y la salud mental (Krause, Carola, Echávarri, Valdés, \& Strasser, 2016); además, puede encontrarse el matiz de la denominada comunicación interpersonal (Flores Mamani, Garcia Tejada, Calsina Ponce, \& Yapuchura Sayco, 2016). 
Si hay una dimensión verbal, ello implica que hay una no-verbal, que a lo largo de los años se consolidó como un campo de estudio inter e intradisciplinar (Matsumoto, Frank, \& Hwang, 2012) en el que se ha puesto atención a diversidad de fenómenos relacionados con: las comunicaciones del entorno, las ciudades y los espacios físicos que transmiten cómo deben comportarse las personas, la distribución de un salón de clases, las mesas y cubiertos en un restaurante; los rastros que dejan las personas en los espacios físicos, una oficina, la habitación de alguien, la disposición de diferentes objetos parecen visibilizar información sobre la persona, sobre sus estados psicológicos y salud mental; y el modo de vestir, que suele dar indicios respecto a rasgos de personalidad.

Hay quienes afirman que del 100\% de toda comunicación, aproximadamente el $7 \%$ es verbal, el resto es no-verbal y que a su vez éste se subdivide en un 38\% de aspectos prosódicos de la voz y el 55\% llega por expresiones faciales, postura, mirada y gestos (Pons, 2017). La clasificación de Matsumoto (2012) destaca el comportamiento no-verbal como aquellos actos individuales que transmiten mensajes. Al respecto, Ekman y Friesen (1969) determinan cinco categorías de comportamiento no-verbal (véase Tabla 1). 


\section{Tabla 1. Categorías de comportamiento no-verbal}

\begin{tabular}{|c|c|}
\hline Categoría & Descripción \\
\hline Emblemas & $\begin{array}{l}\text { Esta categoría es particular en su uso, pues son expresiones que } \\
\text { suelen usarse cuando el entorno impide la comunicación verbal; } \\
\text { son actos no-verbales que tienen una traducción verbal directa. }\end{array}$ \\
\hline Ilustradores & $\begin{array}{l}\text { Estos son actos que dependen del discurso y tienen la función } \\
\text { de plasmar lo que se ha dicho verbalmente. }\end{array}$ \\
\hline Reguladores & $\begin{array}{l}\text { Son actos que regulan y mantienen los turnos en una conver- } \\
\text { sación entre interlocutores. }\end{array}$ \\
\hline Adaptadores & $\begin{array}{l}\text { Son actos que cubren necesidades del cuerpo o realiza acciones } \\
\text { propias del cuerpo que pueden vincularse con estados de ánimo. }\end{array}$ \\
\hline Muestras de afecto & $\begin{array}{l}\text { Son actos exclusivamente vinculados a expresiones faciales que } \\
\text { reflejan estados emocionales. }\end{array}$ \\
\hline
\end{tabular}

Fuente: (Ekman \& Friesen, 1969)

Posteriormente, Ekman y Frisen (1972) se centraron en los movimientos de las manos, conservando algunas de las categorías, pero lo interesante aquí es que los actos de las manos comenzaron a concebirse como un fenómeno único y distinto a otros actos noverbales (véase Tabla 2). Los autores comprenden estos actos según su origen, uso y codificación. 
Tabla 2. Categorías de comportamiento no-verbal asociado al movimiento de las manos

Los emblemas, tienen siempre la intención de comunicar, no obstante, por lo general se realizan en ausencia de discurso y su significado depende del contexto de la conversación, de ese modo, extender el dedo índice y el anular, pueden significar el número dos o amor y paz.

Los ilustradores, se encuentran vinculados al discurso y sólo pueden entenderse con lo dicho, por lo general tiene la intención de comunicar, pero pueden ser automáticos y no hay un diseño consciente de la forma de la mano. Chasquear, tendrá un significado diferente si es acompañado por "tengo una idea" o "se me olvido".

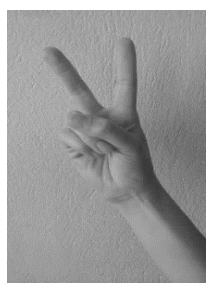

Los adaptadores, no tienen la intención de comunicar y se relacionan con necesidades psicológicas del cuerpo, pero, actos como rascarse las manos puede interpretarse como ansiedad del hablante.
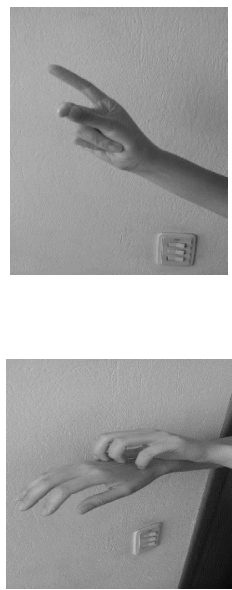

Fuente: (Ekman \& Friesen, 1972)

El origen del acto no-verbal puede ser: por una parte, aprendido en la medida en que existen expresiones que se incorporan al repertorio personal a partir de ver a otros realizarlo en determinados contextos e intencionalidades particulares; por otra parte, los innatismos como las expresiones faciales relacionadas con las emociones básicas. Por lo que se refiere al uso, se ha encontrado que tiene que ver con: 1) la naturaleza de las condiciones externas; 2) cómo el acto se vincula al comportamiento verbal; 3) el nivel de conciencia del acto; 4) la intención de comunicar; 5) la naturaleza de la retroalimentación de la persona que observa el acto; 6) el tipo de información transmitida por el acto. 
Finalmente, la codificación del acto (resumido en la Figura 1) alude a la correspondencia entre la acción y su significado, es decir, un trazo o forma particular de la mano que está vinculado al lenguaje hablado en diferentes formas.

\section{Figura 1. Codificación de los actos no-verbales}

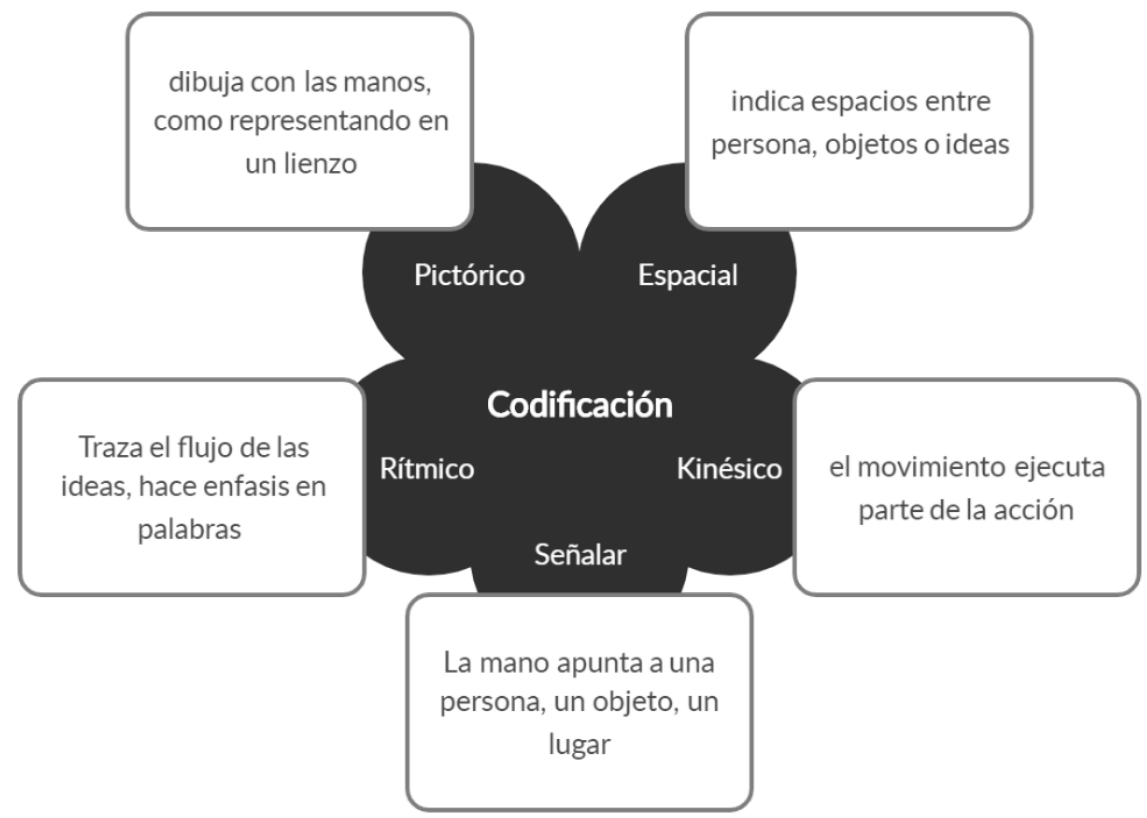

Fuente: elaboración propia

Ekmany Friesen (1972) orientaron sus esfuerzos por las expresiones faciales y las emociones básicas universales; sin embargo, el campo de estudio quedó planteado y retomado por posteriores investigadores. En ese orden de ideas, el interés en la complejidad de los movimientos de brazos y manos favoreció la intención de comprender la cognición y su interacción con el entorno, no solo en lo referido a la cultura y las características de la conversación, sino al espacio físico y a las tareas cotidianas. El cambio de perspectiva lleva a que se abandone el 
término acto no-verbal y se adopte el de gesto. Una de sus principales características es que mano, mente y discurso están profundamente relacionados.

\section{¿Por qué estudiar el gesto?}

Con el desarrollo de nuevas propuestas y miradas sobre la comunicación, creció una perspectiva que superó la dicotomía de lo verbal y lo no-verbal. Kendon (1980) se interesó por las gesticulaciones, que no son más que aquellos movimientos de las manos y los brazos que se hacen junto con el discurso y que se diferencian de otros movimientos del cuerpo por su complejidad. Por lo que se refiere al gesto, se hizo cada vez más evidente que no solo implican una forma concreta de la mano; se sabe que un solo gesto tiene diferentes fases y pueden incluir varios movimientos de la mano (Kendon, 2004). Para el autor, el gesto tiene una función exclusivamente comunicativa, definiéndolos como expresiones manifiestamente deliberadas.

McNeill (1985) afirmó desde sus primeros trabajos que gesto y discurso hacen parte de la misma estructura psicológica, argumentando que el gesto tiene funciones pragmáticas y semánticas paralelas al habla, además de estar sincronizados con unidades lingüísticas. La tesis llevó al autor a considerar que el gesto puede revelar procesos que se encuentran en el pensamiento (McNeill, 1992), inclusive, mano, pensamiento y lenguaje se sostienen en una dialéctica imaginería/ lenguaje que se materializa en gesto/discurso (McNeill, 2005). A razón de su trabajo, se acepta que gesto/discurso es una unidad de análisis robusta y que la mejor manera de estudiarla consiste en no separarlos del lenguaje hablado (McNeill, 2016).

Considérese lo siguiente: si alguien quiere dar indicaciones de una dirección/ubicación en la ciudad, con seguridad esa persona tratará de señalar e ilustrar con las manos una posible ruta, mostrar hacia donde hacer un giro o cuando hay que seguir en línea recta hasta encontrar 
un punto de referencia. Ello sugiere que, la imagen interna es una especie de fotografía en movimiento y que estas acciones se hacen en un espacio y tiempos determinados.

McNeill (1992) propone que, en lugar de tipos de gestos, hay una serie de dimensiones de los movimientos de las manos que ocurren con el discurso. En ese sentido, el autor distingue: gestos icónicos, que son movimientos realizados en el cual el trazo ${ }^{8}$ se sincroniza con el discurso para describir y hacer referencia a aspectos y acciones concretas; gestos metafóricos, que hace referencia a representaciones de ideas abstractas; gestos de ritmo, en la cual las manos se mueven con las "pulsaciones" del discurso. Son esos movimientos que acompañan la velocidad de lo dicho que se caracterizan por ser cíclicos y repetitivos, un claro ejemplo puede observarse en los discursos políticos; 4) gestos cohesivos, que vinculan eventos en el discurso, pero los separa cronológicamente, común en expresiones como: "el año pasado ((Ileva ambas manos a su derecha y las posa en la mesa)) ...pero el próximo ((sus manos se mueven a su izquierda y se mantienen en el aire))"; 5) gestos deícticos, que señalan objetos o eventos concretos del entorno, por ejemplo, señalar al cielo mientras se menciona que va a llover.

Recientemente, hacer distinciones entre tipologías ha dejado de ser centro de atención, en parte debido a que las propuestas por McNeill (2016) no han perdido vigencia; sin embargo, de manera general hay gestos que su principal característica es que son diseñados conscientemente para transmitir un mensaje; otros son automáticos y su intención no es transmitir información (Cooperrider, 2017). Estos últimos, pueden tener funciones asociadas a los turnos en la conversación o al ritmo del discurso.

${ }^{8}$ El gesto tiene tres fases: preparación, trazo y descanso. El trazo (stroke, en inglés) es el que carga el significado del movimiento. 
A continuación, se presenta una serie de extractos que ilustran cómo el gesto puede incidir en el discurso, posibilitando reflexionar sobre cómo el lenguaje es más que el contenido del habla. En el extracto 1, línea 01, la participante representa el número dos de manera sincrónica en el gesto/discurso (Figura 2), según McNeill (2016) aquí no se presenta una redundancia, pues la información es presentada por diferentes canales semióticos; en la línea 02, se puede evidenciar que utiliza cada mano para representar un personaje (Figura 3), y así mismo viso-espacialmente les da un lugar a los sujetos que están inmersos en el discurso, en otras palabras, gestos icónicos que dan lugar a entidades concretas y deícticos ya que señala lugares y los distingue como ubicaciones propias de las entidades en el relato.

\section{Extracto $1^{9}$}

01: *Había * $\underline{\text { dos }}$ *ardillas* *........ gesto*,,,,.,.,.,

Fig. $\quad$ \#fig. 2

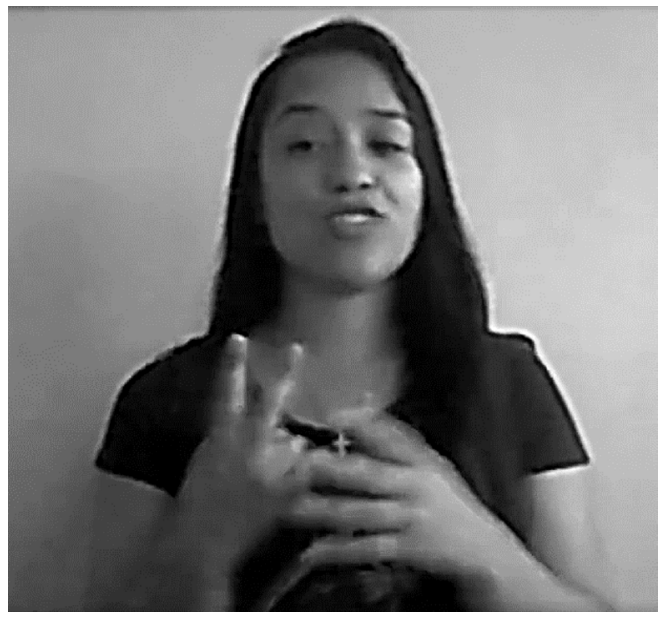

Figura 2. Representa el número dos con los dedos

9 Presentado con la Convención de Lorenza Mondada para la trascripción de interacciones sociales en video. 
02: * * una:::*(x)* ma- (x) una un poco *mayor*que la* otra*

*...*gesto--*,,$*$

Fig. $\quad$ \#fig. 3

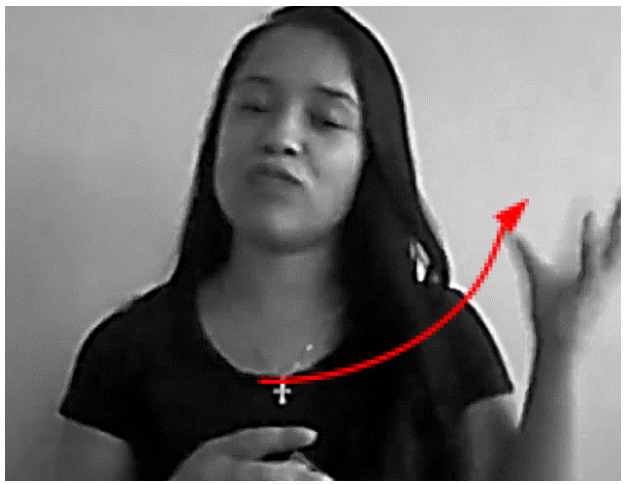

Figura 3. Mano izquierda apunta a un lado

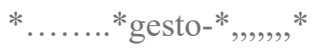

\#fig 4

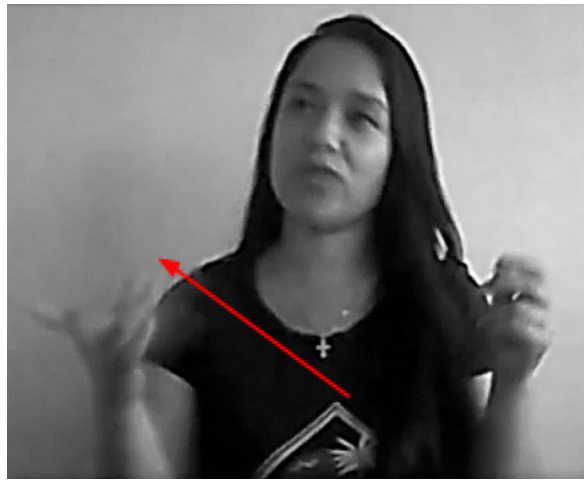

Figura 4. Mano derecha apunta a un lado

Adicional a lo anterior, el extracto permite observar la representación mental sobre la historia relatada, evidenciando que la producción del discurso se enriquece situando los personajes en el contexto de la acción. Nótese que la participante no está teniendo una conversación, a pesar de esto, mueve las manos. Algo similar sucede en una conversación telefónica de solo voz, pese a que no hay contacto visual las personas mueven sus manos. Ello podría indicar que el gesto tiene funciones muy específicas en el hablante.

Ahora bien, si el gesto puede revelar información del pensamiento, en las manos pueden existir claves sobre cómo las personas comprenden su entorno e interactúan con él. Ello lleva a pensar que la forma de la mano se diseña especialmente para transmitir una idea y puede comprenderse de manera explícita desde unidades básicas de significado, lo que se conoce como el punto de desarrollo ${ }^{10}$ planteado por McNeill

${ }^{10}$ En ingles: growth point 
(2000). En el extracto 2, hay dos puntos de desarrollo, el primero en la sincronía con la expresión hacia atrás (Figura 5) en donde la mano derecha señala detrás de su cuerpo y en la palabra hueco (Figura 6), que con la mano derecha representa el vacío y la acción de caer en un mismo trazo.

En relación con lo anterior, el punto de desarrollo de la expresión cae como en un hueco, integra la acción de caer y el vacío en un mismo gesto, aspecto que no podría evidenciarse si solo se tuviera acceso al audio o una transcripción escrita convencional, en la que hueco puede tener una representación difusa y sin movimiento. También sugiere que hablar momento-a-momento implica que en el pensamiento las ideas puedan presentarse de forma integrada, al igual que en las acciones corporeizadas no es posible hacer distinciones semánticas. En el lenguaje hablado, no sería usual encontrar una palabra que represente un vacío que cae.

\section{Extracto 2}

01: la ardilla joven se tropieza como con unos huevos (1) como con un nido

02: y:: *ae*hacia*atrás*, cae como *en *un hueco* *

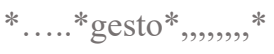

Fig.

\#fig. 5

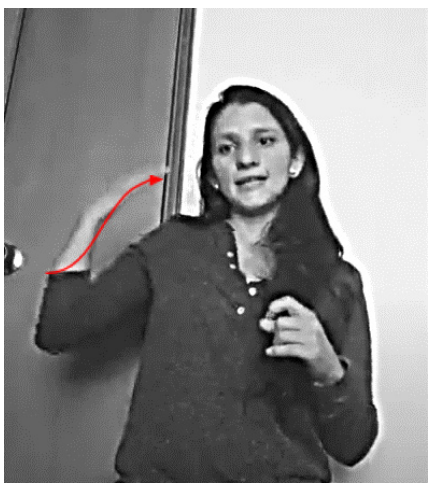

Figura 5. Señala hacia atrás

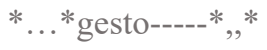

\#fig. 6

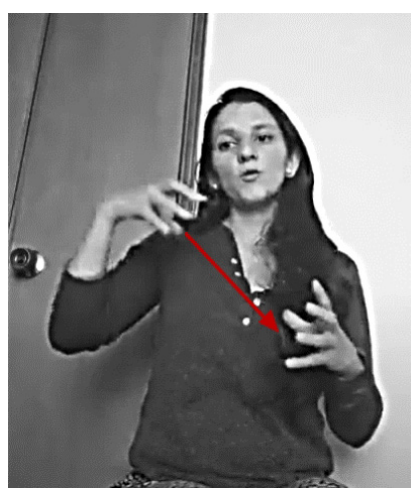

Figura 6. Mano derecha representa vacío y caída, mano izquierda representa el suelo 
En cuanto a los argumentos, no se tardó en explorar implicaciones del gesto en procesos como el aprendizaje, si gesto y discurso son una unidad psicológica, entonces hay aspectos de los movimientos de las manos en particular y en la cognición corporeizada en general, que pueden tener incidencia en la interacción entre estudiantes y docentes. Más aún, las características de la interacción pueden favorecer o no el aprendizaje, lo que es un factor a tener en cuenta si la intención es fortalecer acciones en el aula.

\section{El gesto incide en el aprendizaje: primeras aproximaciones}

Se parte de la idea que el gesto orienta y da forma al pensamiento, además, son actos simbólicos que transmiten significados, lo que implica que hay información y conocimiento contenido en el trazo del gesto (Goldin-Meadow, 2005), lo cual se ha inferido en experimentos que se han hecho en lecciones de matemáticas, y en dónde se ha encontrado que la mano puede mostrar información que no está disponible en el discurso (Goldin-Meadow, Cook, \& Mitchell, 2009). En pocas palabras, el gesto interactúa con el mundo interno y con el externo.

En el extracto 3 línea 01, el gesto funciona en dos sentidos: en términos de señalar cantidad y ayudando a disminuir la velocidad del discurso (Figura 7). En la línea 03, utiliza sus propias gafas para representar una cualidad de uno de los personajes y lo trae al discurso interactuando con un objeto disponible en el mundo físico (Figura 8). Es recurrente que al expresar cantidades en el discurso también se hacen en acciones corporeizadas, lo que puede considerarse como reflejo de un aprendizaje temprano. Contar, sumar y restar suelen aprenderse utilizando los dedos. 


\section{Extracto 3}

01: se puede observar $<\underline{\text { dos }}$ animales en la selva $>*(1) * u n o(1) *$ es*una ardilla (1) hombre Fig. *....*gesto--*,,,*

\#fig. 7

02: y el otro es un oso, <hombre también pero entonces> eh:: es como (2)

03: adulto mayor por las gafas que*te- $<$ *por las* gafas* que tenía puestas $>$

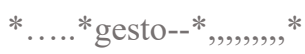

Fig. \#fig. 8

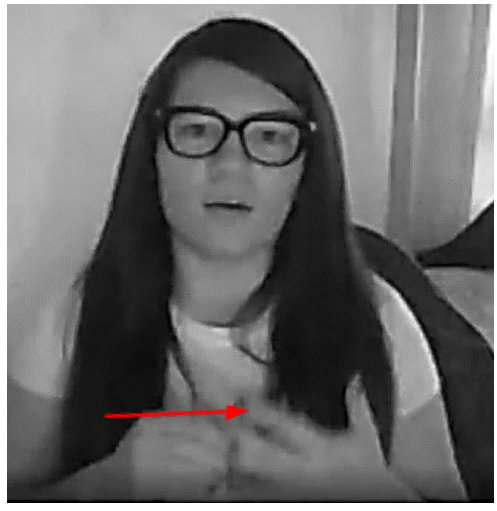

Figura 7. Mano derecha toma un dedo de la mano izquierda

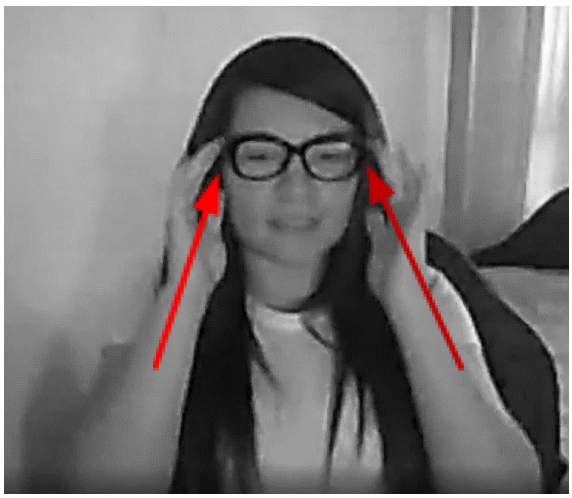

Figura 8. Ajusta las gafas

Se presume que el gesto tiene una función en el aprendizaje, específicamente en relación con mecanismos asociados a la memoria y la percepción (Cook, Mitchell, \& Goldin-Meadow, 2008). Sobre el tema, un estudio quiso observar la incidencia de los gestos de las manos en el aprendizaje de las matemáticas, partiendo de cuestionarse si en la interacción entre instructores y aprendices hay mayor incidencia de la mirada, las expresiones del rostro o los gestos de las manos. La investigación implementó una serie de lecciones por medio de un avatar, el cual fue manipulado para dar la misma lección en dos condiciones: solo habla y habla+gestos, el agente virtual fue programado de modo tal que se mantenía el mismo audio y la misma expresión facial. El estudio 
determinó por medio de pruebas que los niños aprendieron más en la condición de gestos y sacaron mejores puntajes en una prueba posterior (Cook, Friedman, Duggan, Cui, \& Popescu, 2017).

Para ampliar la perspectiva, el gesto es una ventana a la cognición en tanto refleja pensamientos no hablados. La "manipulación" del gesto conlleva a transformar el pensamiento, prueba de ello tiene como base los experimentos realizados con niños en lecciones de matemáticas, el procedimiento consiste en solicitar a los participantes observar las acciones del instructor e imitarlas, por ejemplo, si el instructor señala la operación en una secuencia específica, el niño debía señalar en el mismo orden. El ejercicio de imitar demostró un cambio en la estrategia para abordar el problema (Goldin-Meadow \& Alibali, 2013). Alentar el uso de las manos en la producción de discurso y en los procesos de aprendizaje podría tener aplicaciones en todo contexto educativo, incluso, ser una estrategia pedagógica que invite a usar el lenguaje en todas sus dimensiones (Valderrama Cárdenas, 2019).

El gesto co-ocurre con el lenguaje e influencia el discurso, lo que puede evidenciarse en el extracto 4 línea 01, el hablante representa la acción de desplazarse y define una trayectoria (Figura 9) que coincide con el segmento con mayor fluidez del enunciado, también, crea distancia entre dos eventos de la escena (Figura 10), en la línea 02 el gesto ayuda a hacer énfasis en aspectos referenciales del contexto (Figura 11) y en la 03, representa la acción de correr en movimientos cíclicos de las manos que simulan pisadas (Figura 12). 


\section{Extracto 4}

01: ellas pasaban intentaban *cruzar* de un*lado*a otro, en *un-* (x) en una árbol

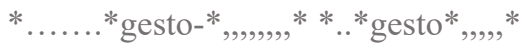

Fig. \#fig. 9 \#fig. 10

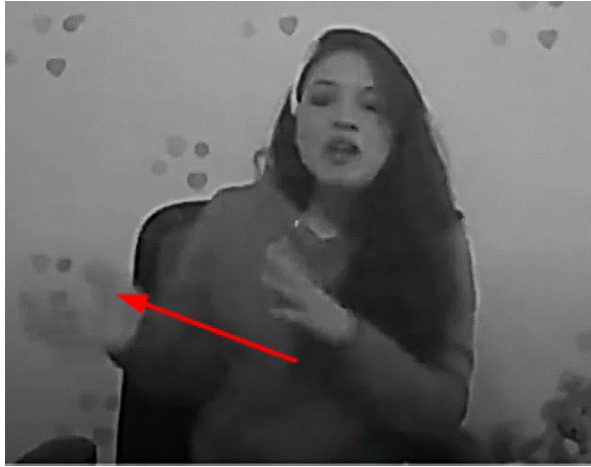

Figura 9. Ambas manos señalan a su derecha

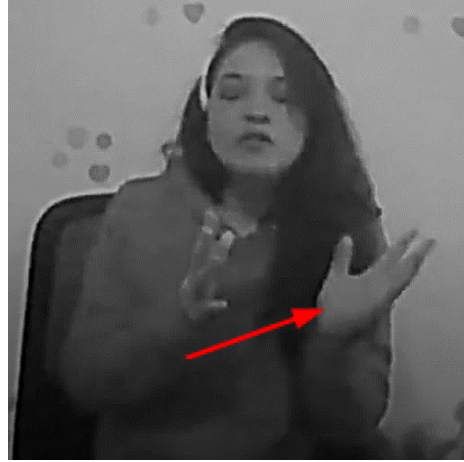

Figura 10. Ambas manos señalan a su izquierda

02: eh:.:.: una le ayudaba a la otra (1) estas pasaro::.n por varias por *varios:: varios* troncos* Fig.

03: varias ramas en *donde* < corrían y*corrían>*

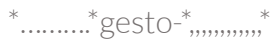

Fig.

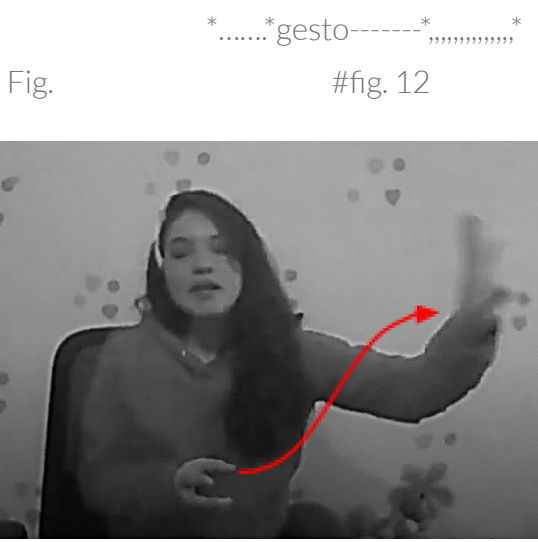

Figura 11. Mano izquierda señala distancia y cantidad

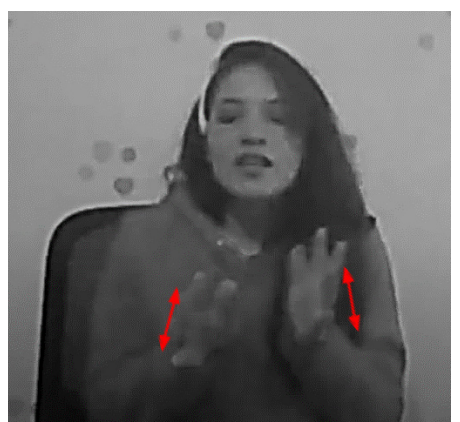

Figura 12. Las manos representan la acción de corre 
Un profesor puede percatarse de cómo sus estudiantes comprenden un concepto o un ejercicio y "manipular" los gestos con la intensión de mejorar la comprensión. Con esto en mente, un estudio se interesó por identificar la atención de los niños en lecciones de matemáticas en operaciones de equivalencia; los experimentadores usaron software de seguimiento de ojos en condiciones de solo habla y habla+gesto. Los niños debian ver videos que explicaban cómo se resolvian las operaciones de equivalencia, para ello, el insctructor hizo previamente videos en donde no hacia gestos y otros en donde señalaba las equivalencias al tiempo que explicaba el ejercicio. En conclusión, la condición de habla+gesto implicó que los estudiantes veian más al instructor y al problema, lo que se relacionó con mejores resultados en una prueba posterior (Wakefield, Novack, Congdon, Franconeri, \& GoldinMeadow, 2018).

Otra conclusión de la investigacion tiene que ver con que los gestos realizados por el instructor ayudaron a guiar la atención de los estudiantes, en otras palabras, se encuentran ventajas en que la atención se centre en la sincronía gesto-discurso. Esto invita a profesores a hacer más acciones corporeizadas en sus clases y ser concientes de ellas, similar al clásico ejercicio de grabarse hablando y darse cuenta de muletillas del habla.

En ese sentido, Cook (2018) describe seis mecanismos que se deben tener en cuenta para el estudio de los gestos en relación con el aprendizaje: 1) los mecanismos perceptuales relacionados con claves en la percepción de los gestos que permiten procesar información lingüística, por lo que se sugiere que el gesto puede ayudar a los hablantes a orientar sus propios procesos perceptivos en apoyo a la produccion del lenguaje; 2) los mecanismos atencionales, que dan cuenta que los gestos son particularmente efectivos para capturar y mantener la atención; 3) mecanismos lingüísticos, que vinculan el gesto con el procesamiento lingüístico, ayudando a codificar y decodificar información; 
4) mecanismos espaciales, centrados en el pensamiento espacial y su incidencia en narrar acontecimientos, haciendo que tenga mayor número de elementos, y mejor capacidad para mantener información relevante; 5) el gesto es evidencia de una cognición situada, extendida y corporeizada. Para finalizar, el autor señala potenciales estudios que permitirán indagar en aspectos que requieren mayor claridad (Tabla 4).

Tabla 4. Mecanismos potenciales en el estudio de gestos

\begin{tabular}{|l|l|}
\hline \multicolumn{1}{|c|}{ Mecanismo } & \multicolumn{1}{|c|}{ Propósitos } \\
\hline Mecanismos perceptuales & $\begin{array}{l}\text { Se deben poner a prueba las características perceptuales } \\
\text { del gesto. }\end{array}$ \\
\hline Mecanismos atencionales & $\begin{array}{l}\text { Se debe comparar diversos tipos de gesto en situaciones } \\
\text { donde pueda evaluarse niveles de atención. }\end{array}$ \\
\hline Mecanismos lingüísticos & Hacer estudios en situaciones naturales \\
\hline Mecanismos espaciales & $\begin{array}{l}\text { Profundizar en las características espaciales de los gestos } \\
\text { de los instructores. }\end{array}$ \\
\hline Mecanismos de memoria & $\begin{array}{l}\text { Identificar en qué procesos asociados de la memoria inci- } \\
\text { den con mayor impacto los gestos. }\end{array}$ \\
\hline $\begin{array}{l}\text { Mecanismos corporeizadosy y } \\
\text { situados }\end{array}$ & $\begin{array}{l}\text { Comprender cómo la cognición corporizada ayuda en } \\
\text { diversos aspectos del pensamiento humano. }\end{array}$ \\
\hline
\end{tabular}

Fuente: (Cook, 2018)

El gesto es fuente de información perceptiva y motora en la comunicación humana (Cook \& Tanenhaus, 2009), tanto que, pueden incidir en las acciones de los oyentes. En un experimento, se le pidió a un grupo de personas resolver el problema de la Torre de Hanoi. La mitad de los participantes lo hicieron con una torre de madera y la otra mitad lo hizo en un computador. A continuación, debían explicar a otra persona cómo resolver el problema. Como resultado se obtuvo que, los participantes que resolvieron el problema con una torre física realizaron movimientos relacionados con levantar una de las piezas y trasladarla; por el contrario, los que lo resolvieron en un computador, realizaron explicaciones que carecían del movimiento de levantar peso y fue más cercano al de arrastrar con el ratón una ficha de un lugar a otro. En 
conclusión, el gesto depende del oyente, más concretamente, depende de a quién se habla.

En ese orden de ideas, el interés contemporáneo en el campo de estudio se relaciona con la sincronía gesto-discurso y su función en diversas tareas de la vida humana. A modo de ejemplo, en el extracto 5, puede observarse que cada uno de los gestos co-expresivos al habla son primariamente procesos de codificación que, son decodificados por el lector (como es su caso) o por el oyente del video. Probablemente el lector se haga una idea del tamaño del árbol o de qué manera llega la otra ardilla: ¿llega saltando? ¿corriendo? ¿caminando?

\section{Extracto 5}

01: Están jugando::*y*correteando::* * po::r* los* $\underline{\text { arboles }}^{*} *$

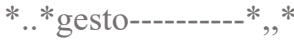

Fig.

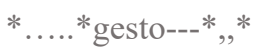

\#fig. 14

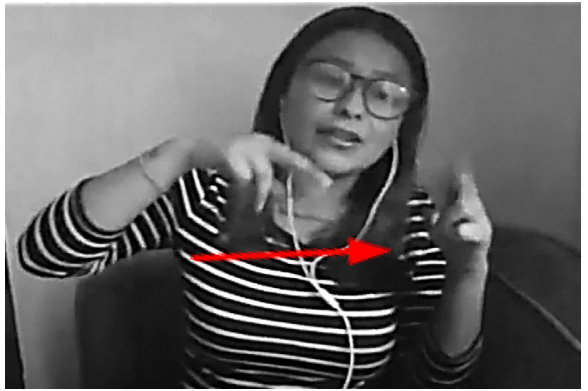

Figura 13. Los dedos representan la acción de caminar

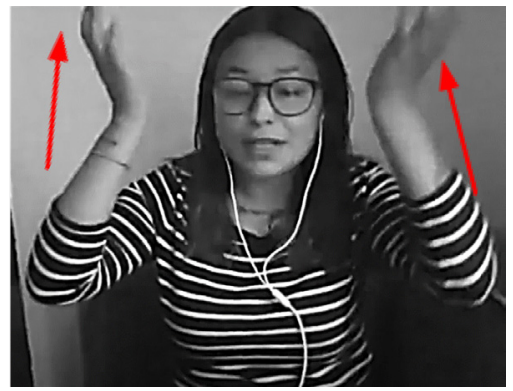

Figura 14. Señala hacia arriba e ilustra la forma del árbol 
02: eh::: luego empieza a dialogar co::n el (x) ardilla mayor anciano que es de color azul *...**gesto*, *, *

Fig. \#fig. 15

03: eh::: después de estar hablando (1) llega eh:: la ardilla (2) pequeña *..*gesto*,*

Fig. \#fig. 16

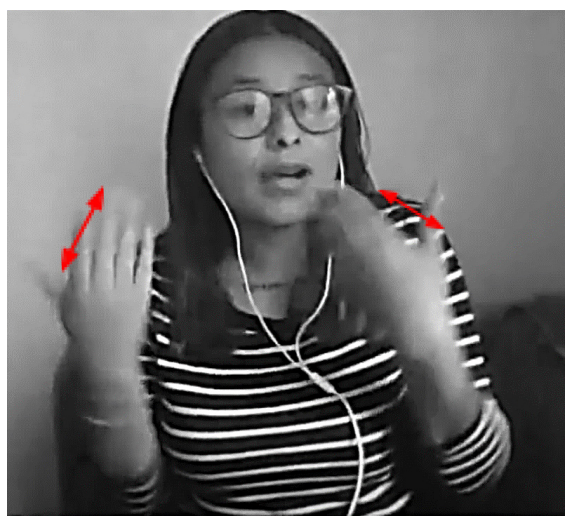

Figura 15. Palmas hacia atrás, movimiento repetitivo de ambas manos hacia adelante y atrás,

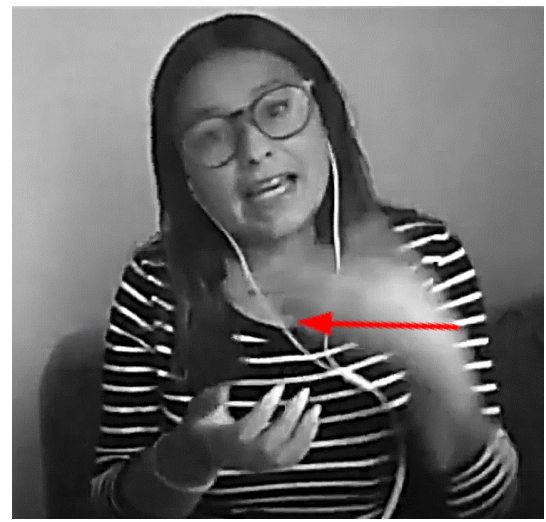

Figura 16. Señala a la derecha

En relación con lo anterior, en la línea 01 se representa una acción (Figura 13) y un espacio (Figura 14) que corresponden al estímulo visual de la historia presentada. Se entiende como una clave en la medida que apoya la memoria para traer representaciones al momento de contar la historia, además, son dos gestos icónicos que expresan imágenes concretas dentro del relato. En la línea 02, dialogar es co-expresado como un intercambio (Figura 15) y el punto de la historia en que los personajes interactúan. En la siguiente línea, resalta la llegada de otro personaje con la mano izquierda (Figura 16).

Algo similar sucede en las lecciones de matemáticas, en dónde se ha comprobado que la sincronía y congruencia son relevantes. Una investigación comparó, por un lado, una lección en donde el profesor 
hacia gestos incongruentes al discurso, por otro lado, otro profesor que los realizaba congruentes, es decir, señalar una suma en el tablero y su procedimiento mientras en el discurso se expresaba lo mismo. Los gestos incongruentes tendieron a confundir a los estudiantes (Ovendale, Brookes, Colletta, \& Davis, 2018). Considérese lo siguiente: en el extracto 5, si el gesto de la línea 02 se realizará de manera sincrónica con la palabra "anciano", en lugar que con la expresión "dialogar", es probable que el sentido fuera confuso y difícil de comprender su función en ese preciso momento.

"Iconic gesture" AND "Learning": en 2020

Tal vez por su característica, los gestos icónicos han captado la atención de diferentes estudios en contextos de aprendizaje. A razón de los anterior, el proyecto se interesó por conocer el estado de la cuestión, por ello, en una búsqueda en la base de datos SCOPUS se introdujeron los criterios "Iconic gesture" AND "Learning". Se obtuvo que, entre 1999 y 2020 hay 64 artículos; 2017 es el año de mayor publicación sobre el tema con diez artículos científicos (véase figura 17).

\section{Figura 17. Publicaciones por año "Iconic gesture" AND Learning}

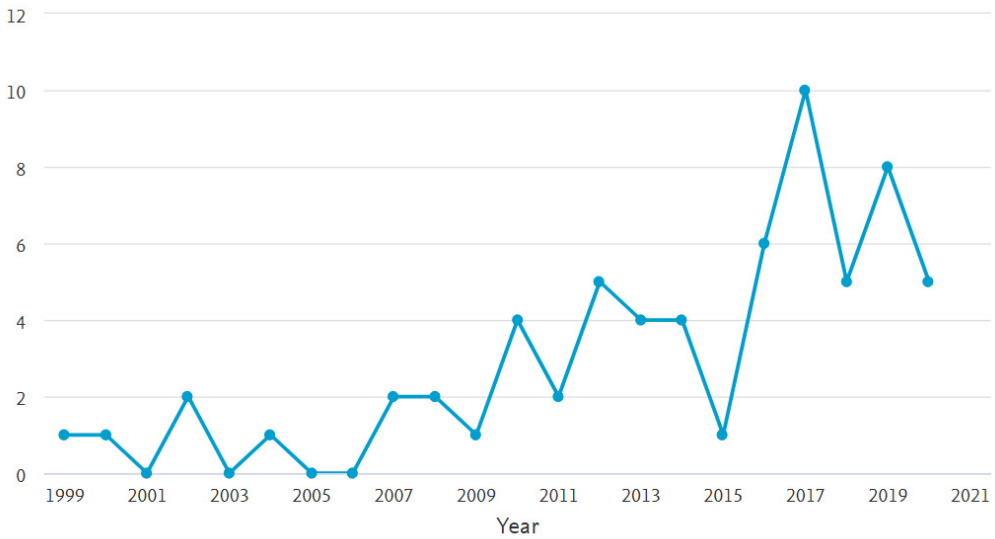

Fuente: Scopus 
En lo que concierne al primer semestre del año 2020, se publicaron cinco investigaciones que relacionan los gestos icónicos con procesos de aprendizaje. Un primer estudio sugiere que los gestos icónicos benefician notoriamente la comprensión narrativa; lo interesante es que este tipo de gesto según refieren los autores, tiene mejores resultados que otros tipos (Dargue \& Sweller, 2020). En un segundo estudio, se preguntan sobre tres variables: edad; dificultad de la tarea; y tipo de gesto. Con el propósito de identificar la incidencia de estas variables en la comprensión narrativa en estudiantes. Se concluyó que la variable que tiene mayor incidencia es el tipo de gesto, además, la estrategia que más beneficia la comprensión de los estudiantes tiene que ver con que el docente realice gestos icónicos que están adecuadamente relacionados con la semántica del discurso (Dargue \& Sweller, 2020).

El tercer estudio se enfoca en el aprendizaje de una lengua extranjera y los beneficios del uso de gestos icónicos. Los autores manifiestan que reproducir los gestos icónicos del instructor hace que se incremente la probabilidad que los estudiantes realicen gestos icónicos espontáneos durante el aprendizaje, el argumento gira alrededor de que la imaginería motora beneficia el aprendizaje de una lengua extranjera, a razón que, la observación en la codificación del profesor mejora la comprensión (Sweller, Shinooka-Phelan, \& Austin, 2020).

Un cuarto estudio se interesa por la adquisición del lenguaje y cómo el gesto icónico tiene una función en el proceso. El autor establece tres funciones; dirigir la atención de los niños en aspectos que llevan información importante y que pueden ser vistos; integrar información de ejemplos para el aprendizaje posterior de los verbos; promueve el conocimiento lingüístico abstracto en la subcategoría de los verbos de locomoción (Aussems, 2020). Un trabajo similar concluye que la habilidad de copiar gestos icónicos se consolida alrededor de los tres años (Bohn, Kordt, Braun, \& Call, 2020). 


\section{Sobre el lenguaje hablado: aprender un idioma extranjero}

Ya se ha visto que el gesto tiene funciones en el aprendizaje en general y en la adquisición del lenguaje en particular; ahora bien, hay indicios que apoyan que el gesto favorece el aprendizaje de un idioma extranjero. Al respecto, no es extraño encontrar estudios que indaguen sobre su influencia. En el mandarín aprender los tonos resulta fonéticamente fundamental para llegar al nivel nativo, con eso en mente, una investigación realiza dos experimentos: el primero a personas adultas hablantes de la primera lengua (L1). El segundo a hablantes de segunda lengua (L2). Los participantes imitaron videos de tonos de mandarín producidos bajo tres condiciones: solo habla, habla+asentimientos de cabeza y habla+gestos. Cada tono tenía una representación gráfica, por ejemplo, un tono podría ser representado por una "w" o por una línea recta, la labor del participante era reproducir el tono y dibujarlo con la mano.

El primer experimento mostró que los tonos de voz no diferían entre las tres condiciones en los hablantes de L1. En el segundo experimento, se evidenció que las condiciones no afectaron la producción de los tonos, no obstante, hubo alguna evidencia de que los gestos con las manos ayudaron en alguno de los tonos. La conclusión de los autores es que no hay evidencia que el gesto haya influenciado en el aprendizaje del tono (Zheng, Hirata, \& Kelly, 2018). Por el contrario, otro estudio que siguió la misma metodología, con la diferencia que implementaron otras acciones de la mano, concluyó que solo algunos tipos de gestos pueden favorecer el aprendizaje de los tonos, en otras palabras, aquellos movimientos que se alinearon con los cambios de tono. El estudio es revelador en la medida en que los autores argumentan que el mapeo de estas características, relacionando movimiento y tono, son modalidades motoras y sensoriales que impulsan el aprendizaje perceptivo (Zhen, Van Hedger, Heald, Goldin-Meadow, \& Tian, 2019). 
Los gestos de ritmo también se han vinculado con el aprendizaje de un idioma extranjero, incluso se ha descubierto que pueden ayudar en la pronunciación (Gluhareva \& Prieto, 2017). Algo que tienen en común diferentes investigaciones es que hay aspectos a tener en cuenta en el profesor y en el estudiante; en los profesores el tipo de gestos que usa o la ausencia de movimientos de las manos. Utilizar solo el habla es la estrategia menos efectiva en el aprendizaje. En los estudiantes parece que el eje central tiene que ver con la atención y con la memoria.

\section{Conclusiones}

Los gestos traen ventajas para hablantes y para oyentes en todo proceso de aprendizaje. Para hablantes, ayuda a organizar el discurso y permite transmitir información que no suele estar disponible en el discurso, también permite dirigir la atención del oyente a eventos del ambiente que son relevantes para la comprensión del mensaje.

La implementación de estrategias corporeizadas en el aula trae beneficios, primero con la propia estructura del discurso del profesor en interacción con el estudiante en contextos de aprendizaje, segundo, la comprensión narrativa se fortalece, lo cual va más allá de la empatía o la relación con el estudiante y tiene que ver con mecanismos multimodales de la comunicación. Las implicaciones se ven directamente relacionadas con el diseño de actividades en donde el estudiante deba usar acciones corporeizadas. Por ejemplo: estimular que señale algún proceso o idea, que represente con las manos su comprensión sobre algo, el que imite alguna acción, hacer pantomimas, entre otros.

Debido a que el campo de estudio es relativamente joven, aún hay temas y aplicaciones por descubrir. Cook (2018) propuso focos que invitan a indagar más sobre la relación que hay entre las acciones corporeizadas en general y los gestos de las manos en particular, con la producción del discurso y los procesos de aprendizaje. Ello implica 
propuestas que tengan en cuenta las interacciones sociales como base de los contextos educativos.

Para finalizar, la comunicación en espacios virtuales sincrónicos es un campo que requiere mayores estudios, aspectos como los turnos en el habla, los actos corporeizados en la comunicación y otras estrategias pueden tener implicaciones en la calidad del aprendizaje. 


\section{Bibliografía}

Aussems, S. (2020). How seeing iconic gestures facilitates action event memory and verb learning in 3-year-old children. Language Acquisition, 27(1), 68-70. doi:https://doi.org/10.1080 /10489223.2019.1624759

Bohn, M., Kordt, C., Braun, M., \& Call, J. T. (2020). Learning novel skills from iconic gestures: A developmental and evolutionary perspective. Psychological Science. doi:https://doi. org/10.1177/0956797620921519

Cook, S. W. (2018). Enhancing learning with hand gestures: Potential mechanisms. In K. Federmeier, The psychology of learning and motivation (pp. 107-133). Cambridge: Elsevier.

Cook, S.W., \& Tanenhaus, M. (2009). Embodied communication: Speakers' gestures affect listeners' actions. Cognition, 113(1), 98-104. doi:https://doi.org/10.1016/j.cognition.2009.06.006

Cook, S. W., Friedman, H. S., Duggan, K. A., Cui, J., \& Popescu, $\checkmark$. (2017). Hand gesture and mathematics learning: lessons from an Avatar. Cognitive science, 41(2), 518-535. doi:https://doi. org/10.1111/cogs.12344

Cook, S. W., Mitchell, Z., \& Goldin-Meadow, S. (2008). Gesturing makes learning last. Cognition, 106(2), 1047-1058. doi:https:// doi.org/10.1016/j.cognition.2007.04.010

Cooperrider, K. (2017). Foreground gesture, background gesture. Gesture, 16(2), 176-202. doi:10.1075/gest.16.2.02coo

Dargue, N., \& Sweller, N. (2020). Learning Stories Through Gesture: Gesture's Effects on Child and Adult Narrative Comprehension. Educational Psychology Review, 32(1), 249276. doi:https://doi.org/10.1007/s10648-019-09505-0 
Dargue, N., \& Sweller, N. (2020). Two hands and a tale: When gestures benefit adult narrative comprehension. Learning and Instruction, 68. doi:https://doi.org/10.1016/j. learninstruc.2020.101331

Ekman, P., \& Friesen, W. (1969). The repertoire of nonverbal behavior: Categories, origins, usage, and coding. Semiotica, 1(1), 49-98. doi:https://doi.org/10.1515/semi.1969.1.1.49

Ekman, P., \& Friesen, W. (1972). Hand movements. Journal of communication, 22(4), 353-374. doi:https://doi. org/10.1111/j.1460-2466.1972.tb00163.x

Flores Mamani, E., Garcia Tejada, M. L., Calsina Ponce, W. C., \& Yapuchura Sayco, A. (2016). as habilidades sociales y la comunicación interpersonal de los estudiantes de la Universidad Nacional del Altiplano-Puno. Comuni@cción, 7(2), 5-14. shorturl. at/sBPR2

Follari, J. E. (2015). El código de transcripción de Gail Jefferson: adaptación para las ciencias sociales. Quaderns de Psicologia,, 17(1), 39-62. doi:https://doi.org/10.5565/rev/qpsicologia.1252

Gluhareva, D., \& Prieto, P. (2017). Training with rhythmic beat gestures benefits $L 2$ pronunciation in discourse-demanding situations. Language Teaching Research, 21(5), 609-631. doi:https://doi.org/10.1177/1362168816651463

Goldin-Meadow, S. (2005). Hearing Gesture: How our hands help us think. Cambridge: The Belknap Press of Harvard University Press.

Goldin-Meadow, S., \& Alibali, M. W. (2013). Gesture's role in speaking, learning, and creating language. Annual review of psychology, 64, 257-283. doi:https://doi.org/10.1146/annurevpsych-113011-143802 
Goldin-Meadow, S., Cook, S. W., \& Mitchell, Z. A. (2009). Gesturing gives children new ideas about math. Psychological Science, 20(3), 267-272. doi:https://doi.org/10.1111/j.14679280.2009.02297.x

Kendon, A. (1980). Gesticulation and Speech: Two Aspects of the process of utrerance. In M. R. Key, The relationship of verbal and nonverbal communication (pp. 207-227). New York: Mouton Publishers.

Kendon, A. (2004). Gesture: Visible action as utterance. Edinburgh: Cambridge University Press.

Krause, M. A., Carola, P., Echávarri, O., Valdés, N., \& Strasser, K. (2016). Comunicación verbal terapéutica en episodios de cambio: un microanálisis comparativo de las formas lingüísticas básicas. Estudios de Psicología, 37(2-3), 514-547. doi:https://doi.org/10 $.1080 / 02109395.2016 .1227575$

Matsumoto, D., Frank, M. G., \& Hwang, H. S. (2012). Nonverbal communication: Science and applications. London: Sage.

McNeill, D. (1985). So you think gestures are nonverbal? Psychological Review, 92(3), 350-371. doi:https://doi. org/10.1037/0033-295X.92.3.350

McNeill, D. (1992). Hand and mind: What gestures reveal about thought. Chicago: University of Chicago press.

McNeill, D. (2005). Hand and Thought. Chicago: University of Chicago Press.

McNeill, D. (2016). Why We Gesture: The surprising role of hand movements in communication. Chicago: Cambridge University Press. 
McNeill, D., \& Duncan, S. (2000). Growth points in thinkingfor-speaking. In D. McNeill, Language and gesture (pp. 141-161). Cambridge: Cambridge University Press.

Mondada, L. (2016). Challenges of multimodality: Language and the body in social interaction. Journal of sociolinguistics, 20(3), 336-366. doi:https://doi.org/10.1111/josl.1_12177

Ovendale, A., Brookes, H., Colletta, J. M., \& Davis, Z. (2018). The role of gestural polysigns and gestural sequences in teaching mathematical concepts: The case of halving. Gesture, 17(1), 128157. doi:https://doi.org/10.1075/gest.00013.ove

Pons, C. (2017). Comunicación no verbal. Barcelona: Kairós.

Ridao Rodrigo, S. (2017). «Es un lector, no un orador»: sobre la tricotomía comunicación verbal, paraverbal y no verbal. Revista Digital de Investigación en Docencia Universitaria, 11(1), 77192. doi:https://dx.doi.org/10.19083/ridu.11.499

Sweller, N., Shinooka-Phelan, A., \& Austin, E. (2020). The effects of observing and producing gestures on Japanese word learning. Acta Psychologica, 207. doi:https://doi.org/10.1016/j. actpsy.2020.103079

Valderrama Cárdenas, J. C. (2019). Por qué y para qué estudiar los gestos. In J. A. Bernate, M. J. Betancourt Jiménez, J. A. Carrillo Villamizar, I. P. Fonseca Franco, M. F. García Celis, W. S. Ortiz Navarrete, ... Urrea Roa, Perspectiva de Transformación Social en la Educación y la Empresa (pp. 73-103). Bucaramanga: Editorial Eidec.

Van Dijk, T. (2000). El discurso como interacción social. Barcelona : Editorial Gedisa. 
Viney, L. (1983). The assessment of psychological states through content analysis of verbal communications. Psychological Bulletin, 94(3), 542-563. doi:https://doi.org/10.1037/00332909.94.3.542

Wakefield, E., Novack, M. A., Congdon, E. L., Franconeri, S., \& Goldin-Meadow, S. (2018). Gesture helps learners learn, but not merely by guiding their visual attention. Developmental science, 21(6). doi:https://doi.org/10.1111/desc.12664

Zhen, A., Van Hedger, S., Heald, S., Goldin-Meadow, S., \& Tian, X. (2019). Manual directional gestures facilitate cross-modal perceptual learning. Cognition, 187, 178-187. doi:https://doi. org/10.1016/j.cognition.2019.03.004

Zheng, A., Hirata, Y., \& Kelly, S. D. (2018). Exploring the effects of imitating hand gestures and head nods on L1 and L2 Mandarin tone production. Journal of Speech, Language, and Hearing Research, 61(9), 2179-2195. doi:https://doi.org/10.1044/2018_ JSLHR-S-17-0481 